\title{
Supracrestal Non-Surgical Therapy in Periodontal Diseases
}

\author{
Khushboo Goel \\ 'Department of Periodontology and Oral Implantology, College of Dental Surgery, B.P. Koirala Institute of Health Sciences, \\ Dharan, Nepal.
}

\section{ABSTRACT}

Periodontal diseases are the complex disease with a dynamic relationship between biofilm and the host immunoinflammatory response. The goal of periodontal therapy is to preserve the natural dentition and increase their longevity by creation of a favorable environment around the teeth. The mainstay to achieve this is by the non-surgical periodontal therapy, followed by surgical and other recent treatment modalities. However, there seems no sure indication to choose amongst them that are clinically significant and offer long term predictability. We report here two cases that had supracrestal defects and were treated with less invasive instrumentation and repeated full mouth scaling and root planing. This avoided a surgical intervention and was more cost-effective in treating moderate to severe young chronic periodontitis patients. The decision for the type of treatment needs to be critically assessed with a better understanding of the outcome, morphology of the defects, and type of teeth involved. Fundamentals like positive reinforcements, compliance and self-performed plaque control will always remain an integral component regardless of nonsurgical or surgical periodontal therapy.

Keywords: debridement; periodontal diseases/therapy; scaling.

\section{INTRODUCTION}

Periodontitis is recognized as an inflammatory disease induced by microbial biofilm and host response. According to data from the World Health Organization, an advanced disease with deep periodontal pockets affects approximately the 10 to $15 \%$ of the adult population worldwide. ${ }^{1}$

Treatment of periodontal diseases depends upon disruption of plaque biofilm and removal of accretions from the diseased tooth and root surfaces. Full mouth scaling and root planing (FMS) combined with oral hygiene efforts and effective compliance provides a favorable environment in supragingival and subgingival niches and helps in arresting the disease progression.

Aimetti $M$ in 2014 mentioned a new classification as supra and subcrestal non-surgical periodontal therapy (NSPT). The supracrestal NSPT included the treatment of gingivitis, nonsurgical coverage of recession-type defects, treatment of suprabony defects and papilla reconstruction techniques. ${ }^{2}$ We present here two cases of supracrestal defects that underwent FMS without antiseptics with the objective to explore the conservative approach in the treatment of moderate to severe chronic periodontitis. We further evaluated whether repeated FMS provides any further gain in attachment levels and reduce the need for surgery.

\section{CASE REPORT 1}

A 22-year-old female presented to the Department of Periodontology and Oral Implantology with the chief complaint of swollen gums since one year. She denied any current and past medical history, adverse habits, allergies, and medications. On examination, her oral hygiene status was poor. There was probing depth (PD) of $7-8 \mathrm{~mm}$ in anterior teeth and attachment loss of $5-6 \mathrm{~mm}$ (Figure 1a). IOPAR (Intra oral peri-apical

Correspondence: Dr. Khushboo Goel, Department of Periodontology and Oral Implantology, College of Dental Surgery, B.P. Koirala Institute of Health Sciences, Dharan, Nepal. Email: khushboo. goel@bpkihs.edu, Phone +977-9842638286. 
radiograph) revealed horizontal bone loss (Figure $1 \mathrm{~b}$, 1c) and a diagnosis of generalized severe chronic periodontitis was made. The patient underwent non-surgical periodontal treatment (NSPT) with a written informed consent obtained. An ultrasonic device (Cavitron, Dentsply, York, PA.) was utilized to remove supragingival and subgingival calculus in a first session, performed by a trained dental hygienist. FMS (without antiseptics) at four weeks and repeated session at three months (Figure 1d) were performed by an experienced periodontist. This procedure was performed with the help of the ultrasonic device, and hand instrument (Gracey curets, Hu-Friedy Instruments, Chicago, IL.) using only light pressure with complete overlapping of instrumentation strokes of limited duration to obtain smooth surfaces within 24 hours. $^{3}$ At six months (Figure 1e) spontaneous repositioning of upper and lower front teeth was observed. Only sites with PD> $3 \mathrm{~mm}$ underwent the second session of subgingival scaling. At nine months (Figure 1f) there was complete resolution of periodontal inflammation. The gingival margins appeared healthy, with no signs of further disease progression. IOPAR (Figure 1 $\mathrm{g}, 1 \mathrm{~h}$ ) revealed repositioning of teeth with no further bone loss. Follow-up sessions at six months and nine months of supragingival scaling were performed by a trained hygienist.

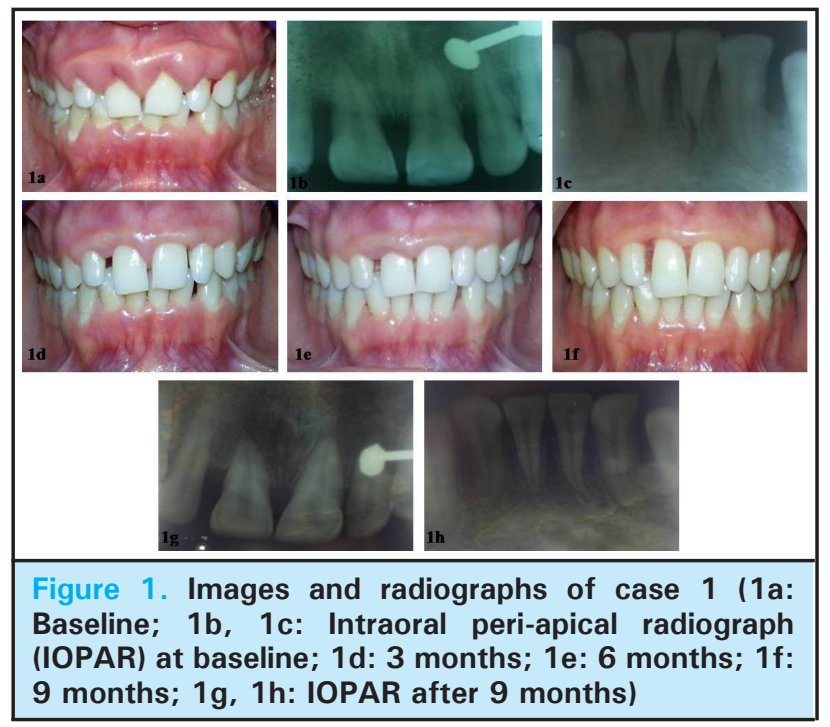

\section{CASE REPORT 2}

A 25-year-old female presented to the Department of Periodontology and Oral Implantology with the chief complaint of bleeding gums since two years. On oral examination, moderate amount of local deposits was present. The gingiva was soft and edematous (Figure 2a) with pseudo PD of 4-5 mm. A diagnosis of generalized chronic gingivitis was made. A similar treatment protocol was followed as the above mentioned case. She was instructed to brush with the modified bass technique and use of interdental aids. At three months there was the partial resolution of edematous gingiva. At six months there was a complete resolution of gingival inflammation with the absence of bleeding on probing (Figure 2b). At nine months an increased stability of gingival margins was achieved (Figure 2c).

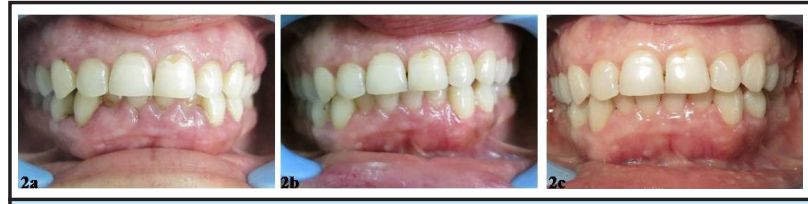

Figure 2. Images of Case 2 (2a: Baseline; 2b: 6 months; 2c: 9 months)

\section{DISCUSSION}

Various longitudinal studies carried out since 1970's prove the effectiveness of NSPT in the treatment of chronic periodontitis. The expected outcomes of NSPT in $\mathrm{PD}$ reduction for moderate and initial deep pockets are $1.29 \mathrm{~mm}$ and $2.16 \mathrm{~mm}$ and attachment gain of 0.55 and $1.29 \mathrm{~mm}$ respectively. ${ }^{4}$ Amongst the various traditional and recent approaches available for NSPT, scaling and root planing (SRP) with personal plaque control is well validated that provides improvement mostly from shrinkage of the soft tissue wall. ${ }^{5,6}$ Comparing SRP with other non-surgical adjuncts, SRP has resulted in $0.5 \mathrm{~mm}$ improvement in attachment level at a moderate level of certainty against 0.2 to $0.6 \mathrm{~mm}$ with SRP and adjuncts. ${ }^{7}$

Enlarged gingiva, sites with $\mathrm{PD}>6 \mathrm{~mm}$ requires further treatment such as repeated subgingival scaling or periodontal surgery. With supracrestal defects involving anterior teeth, after the initial cause-related therapy, both the cases underwent repeated FMS procedure. Recently a systematic review and meta-analysis showed no significant differences in treatment of chronic periodontitis for full mouth disinfection vs. FMS vs. quadrant-SRP. ${ }^{8}$ Extreme care were taken during all sessions to use both hand and ultrasonic instruments with no intentional removal of tooth cementum. Both the cases reflected successful treatment of NSPT in moderate to severe young chronic periodontitis patients. Second session of repeated FMS provided additional benefit by reducing PD and gain in CAL and avoided the need for surgery. Although, better treatment outcomes has been achieved by NSPT for moderate pockets $(\approx 6 \mathrm{~mm})$ and periodontal surgery serves as the gold standard for the treatment of deep periodontal pockets. ${ }^{9}$ Yet, studies that followed patients for three years or more found that, even for deep pockets, NSPT was as effective as surgical treatment and these differences were reduced to less than $0.4 \mathrm{~mm} \cdot{ }^{10}$

Healing in NSPT occurs mostly in six weeks with greatest 
Goel. Supracrestal Non-Surgical Therapy in Periodontal Diseases

at three months and this may continue for additional nine to 12 months or longer. ${ }^{4,11}$ Post-treatment pocket depths in both the cases reduced to $2-3 \mathrm{~mm}$ with the absence of bleeding on probing at follow-up visits at nine months. The findings are similar to the study done by Konig et al in 2008 that showed improvement in clinical periodontal parameters in young adults. ${ }^{12}$ There was also spontaneous repositioning of upper and lower teeth after conventional therapy in case 1 . This might have resulted from wound contraction phenomenon that might have taken place during healing. Studies have questioned repeated subgingival scaling at three months as it is said to provide with no additional benefits as the areas of the root surface that were not reached during the first instrumentation wound not be reached in the second either. ${ }^{13}$ Additionally, it is supposed to cause accumulated traumatic effects to newly immature tissues and may also cause iatrogenic damage to the root surfaces. ${ }^{14}$ There are no clear guideline to choose the treatment modality for moderate to deep pockets that are clinically significant and offer long term predictability. The goal of the outcome to be achieved, the skill of the operator, type of teeth, number and morphology of periodontal defects and finance may indicate the approach to be followed. Nowadays, esthetics plays a pivotal role; therefore a more conservative approach may opt for the health of periodontal tissues. FMS was performed by an experienced periodontist which might have helped in achieving superior results and avoided the need for periodontal surgery. ${ }^{15} \mathrm{~A}$ decreased time devoted to SRP in more recent studies probably accounts for the diminished results compared to the more classic clinical trials. ${ }^{11}$ However, a significant risk factor for NSPT is disease recurrence and persistence of pockets, therefore, positive reinforcements, compliance of patients, home care oral hygiene are keys for long term periodontal maintenance.

\section{Conflicts of Interest: None.}

Consent: JNMA Case Report Consent Form was signed by the patient and the original is attached with the patient chart.

\section{REFERENCES}

1. Petersen PE, Ogawa H. Strengthening the prevention of periodontal disease: the WHO approach. J Periodontol. 2005;76(12):2187-93. [Full Text | DOI | PubMed]

2. Aimetti M. Nonsurgical periodontal treatment. Int J Esthet Dent. 2014;9(2):251-67. [ㅁubMed]

3. Smart GJ, Wilson M, Davies EH, Kieser JB. The assessment of ultrasonic root surface debridement by determination of residual endotoxin levels. J Clin Periodontol. 1990;17(3):174-8.

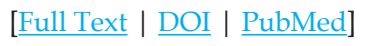

4. Cobb CM. Clinical significance of non-surgical periodontal therapy: an evidence-based perspective of scaling and root planing. J Clin Periodontol. 2002;29(s2):6-16. [Full Text | DOI]

5. Badersten A, Nilveus R, Egelberg J. Effect of nonsurgical periodontal therapy. II. Severely advanced periodontitis. J Clin Periodontol. 1984;11(1):63-76. [Full Text | DOI | PubMed]

6. Ramfjord SP, Caffesse RG, Morrison EC, Hill RW, Kerry GJ, Appleberry EA, et al. 4 modalities of periodontal treatment compared over 5 years. J Clinical Periodontol. 1987;14(8):445-52. [Full Text | DOI | PubMed]

7. Smiley CJ, Tracy SL, Abt E, Michalowicz BS, John MT, Gunsolley J, et al. Systematic review and meta-analysis on the nonsurgical treatment of chronic periodontitis by means of scaling and root planing with or without adjuncts. J Am Dent Assoc. 2015;146(7):508-24. [Full Text | DOI | PubMed]

8. Fang H, Han M, Li QL, Cao CY, Xia R, Zhang ZH. Comparison of full-mouth disinfection and quadrant-wise scaling in the treatment of adult chronic periodontitis: a systematic review and meta-analysis. J Periodontal Res. 2016;51(4):417-30. [Full $\underline{\text { Text }}$ | DOI | PubMed]
9. Heitz-Mayfield LJ, Trombelli L, Heitz F, Needleman I, Moles D. A systematic review of the effect of surgical debridement vs non-surgical debridement for the treatment of chronic periodontitis. J Clin Periodontol. 2002;29(s3):92-102. [․ull Text | DOI | PubMed]

10. Hung HC, Douglass CW. Meta-analysis of the effect of scaling and root planing, surgical treatment and antibiotic therapies on periodontal probing depth and attachment loss. J Clin Periodontol. 2002;29(11):975-86. [ Full Text | DOI | PubMed]

11. Greenstein G. Nonsurgical periodontal therapy in 2000: a literature review. J Am Dent Assoc. 2000;131(11):1580-92. [Full Text $|\underline{\text { DOI }}| \underline{\text { PubMed] }}$

12. Konig J, Schwahn C, Fanghanel J, Plotz J, Hoffmann T, Kocher T. Repeated scaling versus surgery in young adults with generalized advanced periodontitis. J Periodontol. 2008;79(6):1006. [․ㅏll Text $\mid$ DOI | PubMed]

13. Anderson GB, Palmer JA, Bye FL, Smith BA, Caffesse RG. Effectiveness of subgingival scaling and root planing: single versus multiple episodes of instrumentation. J Periodontol. 1996;67(4):367-73. [Full Text | DOI | PubMed]

14. Jenkins WM, Said SH, Radvar M, Kinane DF. Effect of subgingival scaling during supportive therapy. J Clin Periodontol. 2000;27(8):590-6. [Full Text | DOI | PubMed]

15. Brayer WK, Mellonig JT, Dunlap RM, Marinak KW, Carson RE. Scaling and root planing effectiveness: the effect of root surface access and operator experience. J Periodontol. 1989;60(1):67-72. [피l Text $\mid$ DOI | PubMed] 